\title{
Isolation of Cardiomyocytes from Fixed Hearts for Immunocytochemistry and Ploidy Analysis
}

Doğacan Yücel ${ }^{1,2}$, Jacob Solinsky ${ }^{2}$, Jop H. van Berlo ${ }^{1,2,3}$

${ }^{1}$ Department of Integrative Biology and Physiology, University of Minnesota ${ }^{2}$ Lillehei Heart Institute, Department of Medicine, University of Minnesota ${ }^{3}$ Stem Cell Institute, University of Minnesota

*These authors contributed equally

\section{Corresponding Author}

Jop H. van Berlo

jvanberl@umn.edu

\section{Citation}

Yücel, D., Solinsky, J., van Berlo, J.H. Isolation of Cardiomyocytes from Fixed Hearts for Immunocytochemistry and Ploidy Analysis. J. Vis. Exp. (164), e60938, doi:10.3791/60938 (2020).

DOI

URL

$10.3791 / 60938$ jove.com/video/60938

October 7, 2020

\section{Abstract}

The adult mammalian heart is composed of various cell types including cardiomyocytes, endothelial cells and fibroblasts. Since it is difficult to reliably identify nuclei of cardiomyocytes on histological sections, many groups rely on isolating viable cardiomyocytes prior to fixation to perform immunostaining. However, these live cardiomyocyte isolation techniques require optimization to maximize the yield, viability and quality of the samples, with inherent fluctuations from sample to sample despite maximum optimization. Here, we report a reproducible protocol, involving fixation prior to enzymatic digestion of the heart, which leads to maximum yield while preserving the in vivo morphology of individual cardiomyocytes. We further developed an automated analysis platform to determine the number of nuclei and DNA content per nucleus for individual cardiomyocytes. After exposing the chest cavity, the heart was arrested in diastole by perfusion with $60 \mathrm{mM} \mathrm{KCl}$ in PBS. Next, the heart was fixed in 4\% paraformaldehyde (PFA) solution, and then digested with $60 \mathrm{mg} / \mathrm{mL}$ collagenase solution. After digestions, cells were singularized by trituration, and the cardiomyocyte fraction was enriched via differential centrifugation. Isolated cardiomyocytes were stained for Troponin $T$ and $\alpha$-actinin to assess purity of the obtained population. Furthermore, we developed an image analysis platform to determine cardiomyocyte nucleation and ploidy status following DAPI staining. Image based ploidy assessments led to consistent and reproducible results. Thus, with this protocol, it is possible to preserve native morphology of individual cardiomyocytes to allow immunocytochemistry and DNA content analysis while achieving maximum yield.

\section{Introduction}

Heart disease has been the leading cause of death in the majority of western countries for many decades ${ }^{1,2}$. Although many improvements in the treatment of cardiovascular diseases have improved survival, there are currently no treatments that can replace lost cardiomyocytes. Therefore, 
studies related to cardiomyocyte function, proliferation, apoptosis and hypertrophy have been and continue to be a major focus of the scientific community. Since the adult mammalian heart has a very limited regenerative capacity, with an estimated cardiomyocyte renewal rate of less than $1 \%$ per year, it is crucially important to reliably identify cardiomyocyte proliferative events ${ }^{3,4}$. Most strategies that measure proliferative events rely either on staining for incorporated DNA nucleotide analogs to assess previous or current proliferation, or stain for nuclear markers of active proliferation $^{5}$. It is especially important to reliably identify cardiomyocyte proliferative events since the overall number of proliferative cardiomyocytes is so low ${ }^{3,6}$. For example, based on a $1 \%$ renewal rate of endogenous cardiomyocytes per year, one can expect to find between 25 and 50 cardiomyocytes to be proliferative at any given time in the adult mouse heart ${ }^{7,8}$. Any inaccuracies in identification of cardiomyocyte nuclei might lead to false positive results. Therefore, it is critical to reliably identify cardiomyocyte nuclei, which has proven difficult and unreliable from histological sections $^{9}$. Identification of cardiomyocytes is much more accurate from single cells than from tissue sections as it might be difficult to distinguish cardiomyocytes from other cell types even when using markers such as a-actinin, although PCM1 might be a reliable marker of cardiomyocyte nuclei in histological sections ${ }^{10}$.

Current protocols rely on isolating live cardiomyocytes prior to fixation, which is known to cause death of at least $30 \%$ of cardiomyocytes, and might lead to inadvertent selection of specific populations of cardiomyocytes ${ }^{11}$. Furthermore, these protocols are notoriously difficult to optimize to provide reproducible results. Even optimized isolation techniques can typically produce no more than $65 \%$ live, rod-shaped cardiomyocytes with varying yields ${ }^{12}$.

To overcome these issues, we developed a protocol that allows researchers to isolate fixed cardiomyocytes. Since the samples are fixed prior to isolation, the yield is maximized, and in vivo morphology is well preserved. Moreover, with this protocol it is possible to isolate cardiomyocytes from clinical samples, which are typically fixed immediately after procurement. Furthermore, to identify newly generated cardiomyocytes, it is important to measure the nucleation and ploidy status of individual cardiomyocytes, since only diploid cardiomyocytes are typically assumed to be newly formed. Flow cytometry cannot distinguish multinucleation from polyploidy and is a relatively time and resourceintensive protocol. Manual outlining and measurement of nuclei within images is very low-throughput and prone to human bias. Automated quantification of images of fixed, isolated DAPI-stained cardiomyocytes solves both of these problems. Imaging-based determination of nucleation and ploidy distributions can be obtained with a minimum of time and reagents using basic equipment.

\section{Protocol}

All animal experiments were performed conform the National Institutes of Health guidelines and approved by the University of Minnesota Institutional Animal Care and Use Committee (IACUC).

\section{Preparation of the solutions and surgical equipment}

1. Prior to isolation, sterilize the surgical equipment by using $70 \%$ ethanol solution. 
2. Add $2.24 \mathrm{~g}$ of $\mathrm{KCl}$ to $500 \mathrm{~mL}$ phosphate buffered saline (PBS) solution to obtain a final concentration of $60 \mathrm{mM}$. Store $\mathrm{KCl}$-PBS solution at room temperature. Use $3 \mathrm{~mL}$ of $\mathrm{KCl}-\mathrm{PBS}$ solution per mouse.

3. Dilute $32 \%$ paraformaldehyde (PFA) solution with PBS into to obtain final concentration of 4\% PFA. Prepare 10 $\mathrm{mL}$ of $4 \%$ PFA in PBS per mouse. Diluted PFA solution can be stored at $4{ }^{\circ} \mathrm{C}$ for $2-3$ weeks in a glass container. NOTE: Prepared $4 \%$ PFA solution can be stored at -20 ${ }^{\circ} \mathrm{C}$ for longer periods of time.

4. Prepare $1 \mathrm{~mL}$ of collagenase solution per mouse by adding $60 \mathrm{mg}$ of collagenase, type 2 per $1 \mathrm{~mL}$ of PBS.

\section{Perfusion and fixation of the heart}

1. Anesthetize the animal by using $2-5 \%$ isoflurane with an oxygen flow rate of $1 \mathrm{~L} / \mathrm{min}$. Confirm the anesthesia by confirming lack of movement and lower rate of breathing. NOTE: Injecting heparin (100-500 U/kg) before euthanasia can increase the cell quality and yield by preventing blood clots, thereby allowing for more efficient perfusion of the heart with fixative.

2. Euthanize the animal according to approved methodologies.

NOTE: We followed the American Veterinary Medical Association guidelines for the euthanasia of animals, and obtained local IACUC approval for euthanasia.

3. Place the euthanized animal in supine position, and tape down extended limbs.

4. Cut through the chest to expose the heart using blunt-end scissors. Cut descending aorta and inferior caval vein.

5. Perfuse the heart by injecting $3 \mathrm{~mL}$ of $\mathrm{KCl}-\mathrm{PBS}$ solution through the left ventricle with a flow rate of $3 \mathrm{~mL} / \mathrm{min}$ using a peristaltic pump attached to an infusion set with a 23
$\mathrm{G}$ butterfly needle (26 $\mathrm{G}$ for neonates). Make sure not to pierce through the septum.

NOTE: Alternatively, use a needle attached to a syringe to inject solutions.

6. Perfuse the heart by injecting $10 \mathrm{~mL}$ of $4 \%$ PFA solution for $10 \mathrm{~min}$ using a peristaltic pump at a rate of $1 \mathrm{~mL} / \mathrm{min}$.

7. Remove the whole heart using scissors. After removing the heart, it is possible to isolate a specific region of the heart by incising. Place the heart, or a segment of it in a $1.5 \mathrm{~mL}$ microcentrifuge tube containing $1 \mathrm{~mL}$ of $4 \%$ PFA solution. Incubate the heart on rocker at room temperature with rocking speed between 20-30 rpm for 1 h.

\section{Isolation of fixed cardiomyocytes}

1. Place the heart in a Petri dish containing PBS solution. Squeeze the heart to get rid of any PFA remaining in ventricles, and wash in PBS.

2. Put the fixed heart into a new $1.5 \mathrm{~mL}$ microcentrifuge tube containing collagenase solution $(60 \mathrm{mg} / \mathrm{mL})$. Place the tube on rocker (20-30 rpm) at $37^{\circ} \mathrm{C}$ for overnight incubation.

NOTE: Extend the incubation time up to 1 week and replenish the collagenase solution every two days to reduce the possible variation in yield if hearts are anticipated to be fibrotic, which might require longer time of collagenase digestion to digest extracellular collagen.

3. Put collagenase solution and the heart into a $35 \mathrm{~mm}$ Petri dish. Dissociate the heart into $1 \mathrm{~mm}$ pieces by using forceps or scissors.

4. Use a transfer pipette to further triturate the dissociated tissue for $2 \mathrm{~min}$. If tissue particles still remain in the dish, use a transfer pipette with narrower opening and continue 
trituration. Continue until the majority of the tissue is broken down.

NOTE: Over trituration causes individual cardiomyocytes to break. Make sure not to over triturate by checking regularly under a microscope.

5. Place a $200-600 \mu \mathrm{m}$ nylon mesh over opening of $15 \mathrm{~mL}$ centrifuge tube.

NOTE: For hypertrophied cardiomyocytes, it is recommended to use $400 \mu \mathrm{m}$ nylon mesh instead of 200 $\mu \mathrm{m}$.

6. Add $5 \mathrm{~mL}$ of PBS to the Petri dish containing dissociated cells and filter the solution through nylon mesh, including tissue particles. Wash the nylon mesh by passing additional $4 \mathrm{~mL}$ PBS.

7. Centrifuge the filtered solution at $10-100 \times g$ for $1 \mathrm{~min}$.

NOTE: $100 \times g$ centrifugation will not yield $100 \%$ pure cardiomyocyte population, and some non-cardiomyocyte cells are likely to be included.

8. Discard the supernatant unless one wants to stain/ evaluate non-cardiomyocytes cardiac cells as well. Resuspend the pellet in $10 \mathrm{~mL}$ PBS prior to staining.

\section{Staining cardiomyocytes}

1. Collect the cells by centrifugation at $100 \times g$ for $1 \mathrm{~min}$ and add $5 \mathrm{~mL}$ of permeabilization solution (e.g., $0.5 \%$ Triton $\mathrm{X}-100$ in PBS). Incubate for $20 \mathrm{~min}$ at room temperature on rocker.

NOTE: For steps 4.1, 4.2 and 4.4 use $15 \mathrm{~mL}$ centrifuge tubes as it is easier to remove the supernatant without disturbing the cell pellet compared to $1.5 \mathrm{~mL}$ microcentrifuge tubes.

2. Collect the cells by centrifugation at $100 \times g$ for $1 \mathrm{~min}$, add $5 \mathrm{~mL}$ of blocking buffer (e.g., 3\% bovine serum albumin [BSA] in PBS) and incubate for $30 \mathrm{~min}$ at room temperature on a rocker.

3. Collect the cells by centrifugation at $100 \times g$ for $1 \mathrm{~min}$ and add $1 \mathrm{~mL}$ of primary antibody solution (in PBS) with the appropriate dilution ratio. Transfer the solution into 1.5 $\mathrm{mL}$ microcentrifuge tube and incubate cardiomyocytes in primary antibody solution under optimized conditions (e.g., $4{ }^{\circ} \mathrm{C}$ overnight).

4. Transfer cardiomyocytes with primary antibody solution to a $15 \mathrm{~mL}$ centrifuge tube and add $9 \mathrm{~mL}$ of PBS. Incubate the cardiomyocytes for $10 \mathrm{~min}$ at room temperature on a rocker.

5. Collect the cells by centrifugation at $100 \times g$ for $1 \mathrm{~min}$ and add $10 \mathrm{~mL}$ of PBS. Incubate the cardiomyocytes for 10 min at room temperature on a rocker. Repeat this step once more.

6. Collect the cells by centrifugation at $100 \times g$ for 1 min and add the secondary antibody solution containing DAPI. Incubate for $30 \mathrm{~min}$ at room temperature on a rocker, followed by repeating step 4.5 twice to wash cardiomyocytes.

7. Place the cells either on coverslips or microscopecompatible plates and proceed with imaging.

NOTE: Images included in the manuscript were taken with 10x and 40x objectives. Lasers used were: $405 \mathrm{~nm}$ for DAPI, $561 \mathrm{~nm}$ for Alpha actinin and $640 \mathrm{~nm}$ for Edu.

\section{Setup imaging software}

NOTE: Follow along with these steps using Supplementary File 1-SoftwareScreenshots.pdf.

1. Download the Fiji distribution of ImageJ. 
2. Open Fiji. Click on Help > Update... > Manage Update Sites. Check the "IJPB-plugins" and "Biomedgroup" update sites to download the dependencies plugins Ellipse Split and Morpholibj.

3. Click Close. Fiji should begin downloading the dependencies. Restart Fiji when finished.

4. Download Rstudio and open it.

5. Copy install.packages(c("ggplot2", "autothresholdr", "dplyr", "purrr", "jsonlite", "shiny")) into R console's command line and press the Enter key. Type "y" in response to all prompts to install all $\mathrm{R}$ dependencies (Screenshot 1 in Supplementary File 1).

\section{Image quantification}

1. Open Fiji and drag "AnalyzeNucleation.py" (supplied as a supplementary code file) into Fiji's status bar. This will open a script-editing window. Click Run in the lower left corner to begin it (Screenshot 2 in Supplementary File 1).

2. A dialog box will pop up (Supplementary File 1: Screenshot 3), asking for the location of the output data directory. All analysis data, figures, and other data used by this software will be stored in this folder. Another, larger dialog box will pop up, displaying all image analysis settings (Supplementary File 1: Screenshot 4).

1. Select location of directory containing images to be analyzed.

2. Enter the image filename format using regular expressions. Enter the image filename format, indicating which parts of the filename correspond to row, column, channel, and (optionally) site within braces, using regular expressions. Do not put spaces within the braces. Surround variable parts of the filename format in braces \{\} . The way files are saved depends on the imaging software, and this step will retrieve relevant information from the image filename. NOTE: For example, the format string

r" Plate 1-(?P<row>[A-Za-Z]+)(?P<column>[0-9]+)-(? $\mathrm{P}<$ channel $>[\mathrm{A}-\mathrm{Za}-\mathrm{z}]+)$. .tif"

describes a filename that starts with "Plate 1-", which is followed by one or more alphabetical letters indicating the row, which is followed by one or more digits indicating the column, which is followed by "-", which is followed by one or more letters indicating the channel, which is followed by ".tif". The letters inside the angle brackets like "<>" are variable names and are automatically copied into the data when it is collected. One of the variable names must be "<channel>"

3. Indicate the name of channels in which the nuclear stain is visible and where the cardiomyocytes are visible. These names must be exactly as they are in the part matched by the "<channel>" variable in the regular expression filenames.

4. Indicate how the images should be grouped using comma-separated variable names. All of the images within a given group will be opened and analyzed in one batch. For example, if the images are divided into sets for each well, and there is a well for each unique combination of row and column, then write "row, column" in this field.

NOTE: These grouping variables must be a subset of the variables used in the format string. Do not use "channel" as a grouping variable, this will separate corresponding channel images from each other.

5. Indicate whether or not images are stitched together in one well image or are separate for each site. In 
the former case, site should not be indicated in the filename format string.

6. Choose which thresholding method to use to separate nuclei from the background. All of Fiji's standard thresholding methods are available. Test different thresholding methods to determine which works best for the image set. In this example, choose the Otsu method.

7. Indicate whether or not the threshold should be recalculated for each site image or if the same threshold should be used for every image in the group. Indicate whether the cardiomyocyte images are brightfield or use a fluorescent marker.

8. Indicate the cardiomyocyte thresholding method. If brightfield was chosen in the previous step, this thresholding method will be applied to edge-filtered brightfield images. Indicate whether or not the threshold should be recalculated for each site image or if the same threshold should be used for every image in the group.

9. Indicate the number of rows of site images that cover each well. Indicate the number of columns of site images that cover each well. Indicate the minimum area of nuclei in pixels. Use a generously low minimum size, a higher and more precise threshold will be calculated in the analysis step. Indicate the minimum area of cardiomyocytes.

10. After choosing the desired settings click OK.

3. Images resembling those found in Figure $\mathbf{3}$ and Figure $\mathbf{4}$ will appear on the screen, showing the different stages of the analysis pipeline. Inspect these images to ensure that thresholding and segmentation are occurring properly.
4. The selected results folder should now be filled with analysis data (Supplementary File 1: Screenshot 5). Files other than analysis data can safely be saved in this folder as long as their names do not begin with "cm_", "nuclei_", or "nucleilink_".

\section{Data analysis}

NOTE: The csv files that are produced can be analyzed manually. Each analyzed image subset produces a triplet of csv files named "nuclei(metadata).csv", "nucleilink(metadata).csv", and "cardiomyocytes(metadata),csv", where (metadata) is replaced with a sequence of name-value pairs of the form "_(name)=(value)", where (name) and (value) are sequences of alphanumeric characters derived from strings matched in the regular expression given earlier. (For example, if row and column were indicated in the filenames then strings like "_row=F" and "_column=8" will be present). The unnamed leftmost column of each nuclei and nucleilink file is a nucleus ID number. The "Min" column of the nucleilink file is the id of the cardiomyocyte that contained said nucleus wholly or 0 otherwise. The "Max" column of the nuclei is the ID of the highest-numbered cardiomyocyte that contained said nucleus in part, or 0 otherwise. The "Mean" column of the cardiomyocytes file is the cardiomyocyte id number.

1. Open "AnalyzeMultinucleatedServer.R" in Rstudio (provided as supplementary code file).

2. At the top of this file is a variable named "folderName". Next to it is a filepath. In here, type the path to the output data folder selected in the last step, without the final slash (Supplementary File 1: Screenshot 6).

3. In the upper left corner of the script editing window there should be a green arrow labeled Run App. Click this 
arrow. It may take some time for the data to load and for the app to pop up.

4. Initially, three gating graphs will be visible, one to indicate the minimum valid nuclear area threshold, one to indicate the minimum valid nuclear mean intensity threshold, and one to indicate the maximum valid minimum feret's diameter for cardiomyocytes. Use the sliders to set these thresholds (Supplementary File 1: Screenshot 7).

NOTE: In each of these graphs, a large, broad peak corresponding to valid nuclei or cardiomyocytes should be present, flanked by broad tails representing debris or erroneous segmented cardiomyocytes. Use the thresholds to cut one tail of each of the peaks off.

5. Scroll down. Click the button Apply Selected Thresholds (bottom of Supplementary File 1: Screenshot 7).

6. Click the button Plot Intensity Distribution. This will render plot of the nuclear intensity distribution of both the entire sample and separate subplots for each grouping variable.

NOTE: For example, if <row> and <column> grouping variables were entered into the regular expression in the Fiji dialog, plots indicating the intensity distribution by row and by column will appear here (Supplementary File 1: Screenshot 8). If illumination and staining conditions were constant across the different parts of the sample, these plots should all clearly show two intensity peaks, a dimmer, taller one for the diploid nuclei and a brighter, shorter one for the tetraploid nuclei.

7. Intrasample variation will result in this pattern not being visible in the whole-sample plot and there being great variety in the location of the diploid and tetraploid peaks by row, column, or other grouping variable. In the latter case, scroll down check the checkbox Normalize Separately by group to account for this variation (Supplementary File 1: Screenshot 9).

8. Click the button Calculate Ploidy (Supplementary File 1: Screenshot 9). Click the button Plot Estimated Ploidy Distribution. Graphs will appear in the empty windows to the right. In the normalized whole-sample graph, the twopeak pattern should be visible if it wasn't before.

9. Select thresholds to isolate the diploid and tetraploid peaks from both each other and outliers using the sliders (Supplementary File 1: Screenshot 9). Scroll down. Click the button Calculate Ploidy and Nucleation (Supplementary File 1: Screenshot 10).

10. Click the button Plot and Save Into Results Folder. The plot saved into the selected results folder will also appear in this interactive window (Supplementary File 1: Screenshot 10).

\section{Representative Results}

Cardiomyocytes were isolated according to the protocol described above. Using this method, we typically get uniformly singularized cardiomyocytes that are relatively pure without contaminating non-cardiomyocyte cells (Figure 1A). Cardiomyocytes are easily identified under bright field microscopy due to their characteristic size and birefringence. This technique is easy to implement and provides consistent results from different isolations with comparable cardiomyocyte yields and quality (Figure 1B). Isolated cardiomyocytes can be stored at $4{ }^{\circ} \mathrm{C}$ for several weeks before further use. 
Cardiomyocytes that were isolated according to the above protocol can be used for various downstream applications, such as measuring cardiomyocyte size, cardiomyocyte ploidy and immunocytochemistry. As a representative result, we show that cardiomyocytes isolated according to this protocol can be stained using antibodies and fluorochromeconjugated azides for click chemistry to detect localization of specific proteins or to detect cardiomyocyte DNA replication, respectively. For example, we stained cardiomyocytes with antibodies recognizing $\alpha$-actinin to show the characteristic z-line staining pattern of sarcomeres (Figure 2A). In a separate experiment, we administered the thymidine analog 5-Ethynyl-2'-deoxyuridine (EdU) to mice before isolating fixed cardiomyocytes. After cardiomyocyte isolation, we stained for incorporated EdU using standard protocols ${ }^{13}$, and were able to detect cardiomyocytes that had undergone $S$ phase in either mononucleated, binucleated and trinucleated cardiomyocytes (Figure 2B).
To further expand the utility of the isolation method, we developed a pipeline that allows quantification of cardiomyocyte ploidy based on integrated DNA staining. To be able to measure ploidy status of cells or nuclei, we needed to segment nuclei and cardiomyocytes. Figure $\mathbf{3}$ shows a representation of the strategy we used to identify individual nuclei. First, the original image DNA stained image (Figure 3A) is thresholded based on intensity (Figure 3B). Here, we used DAPI to stain for DNA, but any other nuclear dye that shows a linear correlation with DNA content would work. The program allows for any of Fiji's intensity thresholding methods to be chosen, but in this example Otsu's method was used. Nuclear masks that are touching the edge of the image or are smaller than the specified minimum pixel area threshold are excluded. Then, ellipses are fit to the nuclear masks, segmenting individual nuclei. Figure $\mathbf{3 C}$ shows these ellipses overlaid on the original image. Next, holes are filled in the masks, and the pixels of the image are then partitioned into territories based on which ellipse they are most proximal to (Figure 3D). The borders of these territories are then used to draw lines through nuclear clusters, finishing the nuclear segmentation process (Figure $\mathbf{3 E}$ ). 
The next step involves detection of cardiomyocytes. For cardiomyocyte images that are obtained based on fluorescently stained cells (Figure 4A), the process is very similar to that for nuclei. The image is thresholded based on an intensity value calculated by the selected thresholding method, in this case the triangle method. Identified cardiomyocyte masks that are touching the boundary of the image or are below a certain size are excluded and holes are filled in the masks to provide properly segmented cardiomyocytes (Figure 4B). Because cardiomyocytes have a more irregular shape than nuclei, no attempt is made to segment cardiomyocyte clusters. Instead, these clusters are excluded based on their high minimum Feret's diameter during the analysis step. Segmentation from bright field images proceeds slightly differently. First, the original bright field image (Figure 4C) is processed with a Sobel edge filter. This filter calculates the absolute value of the gradient of each pixel within the image. Pixels in regions with rapid changes receive high values and pixels in smooth regions of the image receive low values. This edge-filtered image is then thresholded by intensity, using the Triangle method, resulting in masked cardiomyocytes (Figure 4D). These highly irregular masks are then smoothed and linked together via morphological closing using a circle with a radius of 2 pixels, which fills in all white regions in the image where the circle cannot fit without overlapping a black region (Figure 4E). Finally, holes in the masks are filled, regions touching the border are excluded, and small particles are removed, finishing the cardiomyocyte segmentation process (Figure 4F).

Using the outlined segmentation strategy, we can then determine the nucleation status of individual cardiomyocytes. Using this approach, we determined the nucleation status of cardiomyocytes isolated from hearts of outbred CD-1 mice at early postnatal time-points. Hearts of newborn mice (first day of life) showed that the majority of cardiomyocytes at that point are mononucleated (Figure 5: neonatal). This high frequency of mononucleated cardiomyocytes is much lower in juvenile mice (2-week old), where mononucleated cardiomyocytes make up about $25 \%$ of the total cardiomyocyte population (Figure 5: juvenile). Finally, we can measure the ploidy status of individual nuclei within cardiomyocytes, and determine whether they are diploid or tetraploid. These results show higher frequency of tetraploid nuclei in adolescent mice (Figure 6). 
A

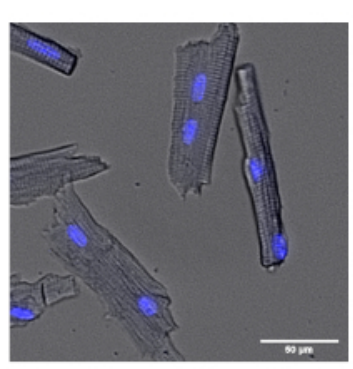

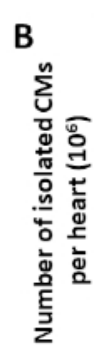

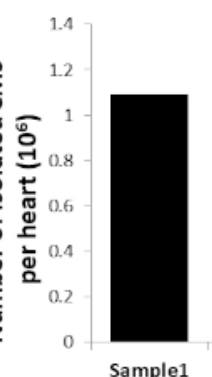

Sample1

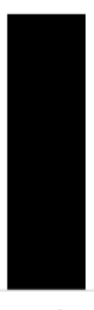

Sample2

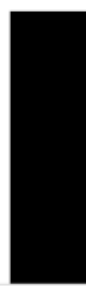

Sample3

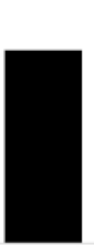

Sample4 Sample5

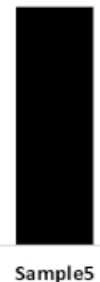

Figure 1: Efficiency of cardiomyocyte isolation after fixation. (A) Representative image of isolated cardiomyocytes stained with DAPI to show nuclei. (DAPI (blue), Brightfield (gray)) (B) Yield of cardiomyocytes isolated from different mice at 3 months of age. Scale bars $=50 \mu \mathrm{m}$. Please click here to view a larger version of this figure.

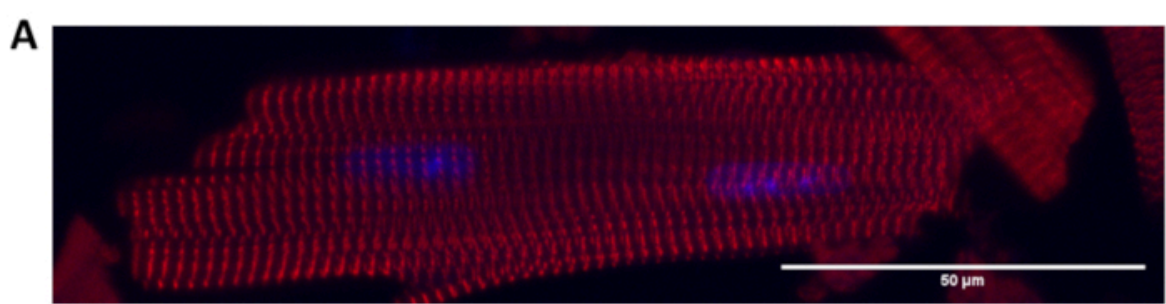

B
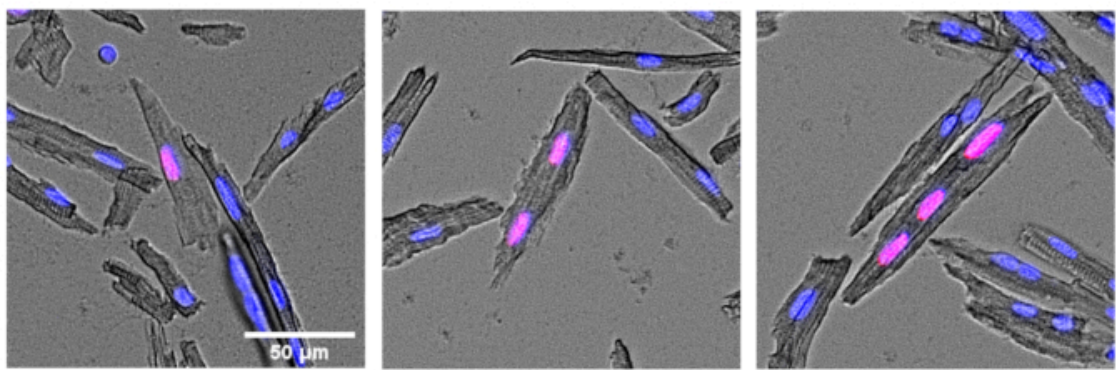

Figure 2: Immunocytochemistry of isolated cardiomyocytes. (A) Representative image of cardiomyocytes stained for $\alpha$-actinin ( $\alpha$-actinin (red) and DAPI (blue)). (B) Cardiomyocytes stained for incorporated EdU (red) and DAPI (blue). Representative cardiomyocytes that are mononucleated (left), binucleated (middle) and trinucleated (right) and EdU positive are shown. Scale bars $=50 \mu \mathrm{m}$. Please click here to view a larger version of this figure. 


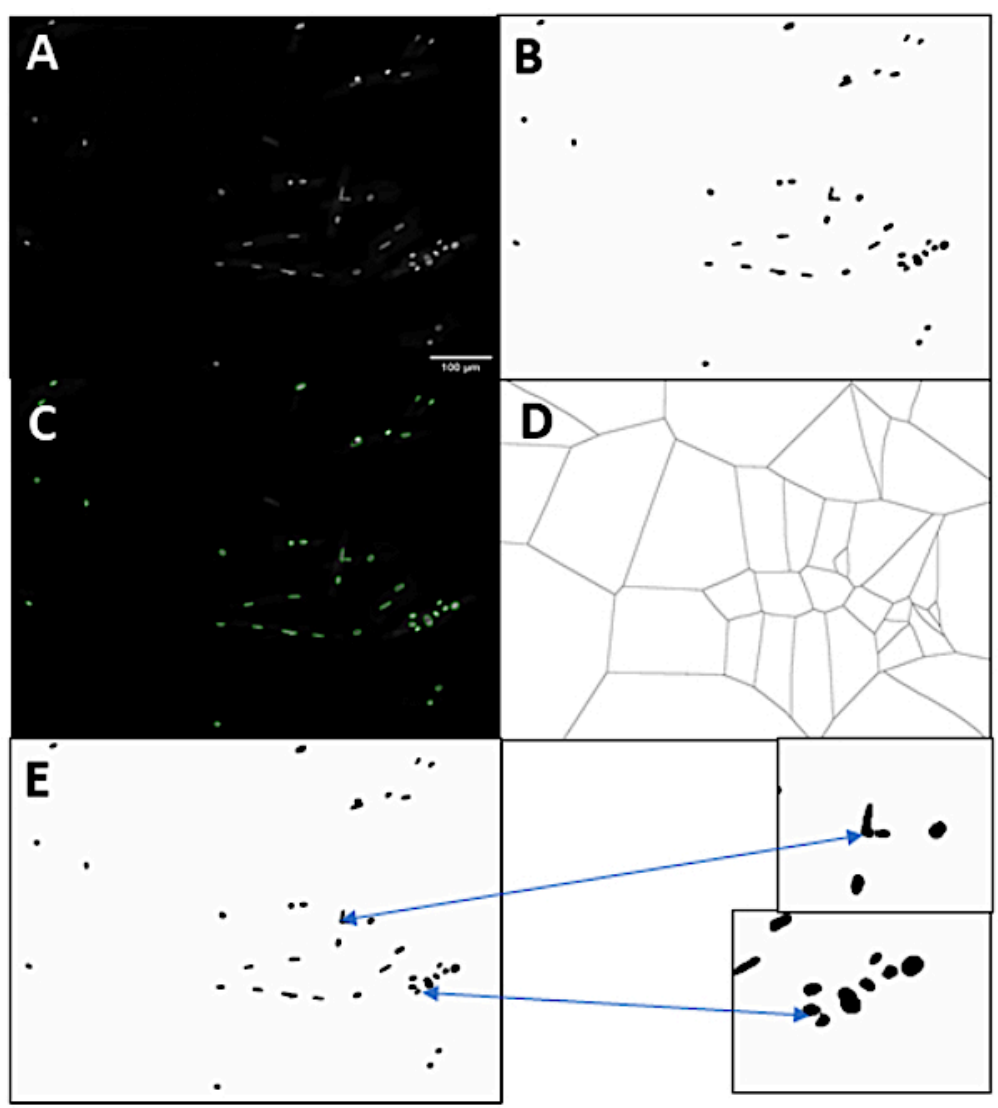

Figure 3: Strategy for nuclear segmentation. (A) Original DAPI channel image. (B) Thresholded image (in this example, Otsu's method was used). (C) Masks that were identified from the thresholded images overlaid on the original DAPI stained image. (D) Voronoi tessellation based on nuclear masks. (E) Final segmented nuclei, with split clusters highlighted. Scale bars $=100 \mu \mathrm{m}$. Please click here to view a larger version of this figure. 


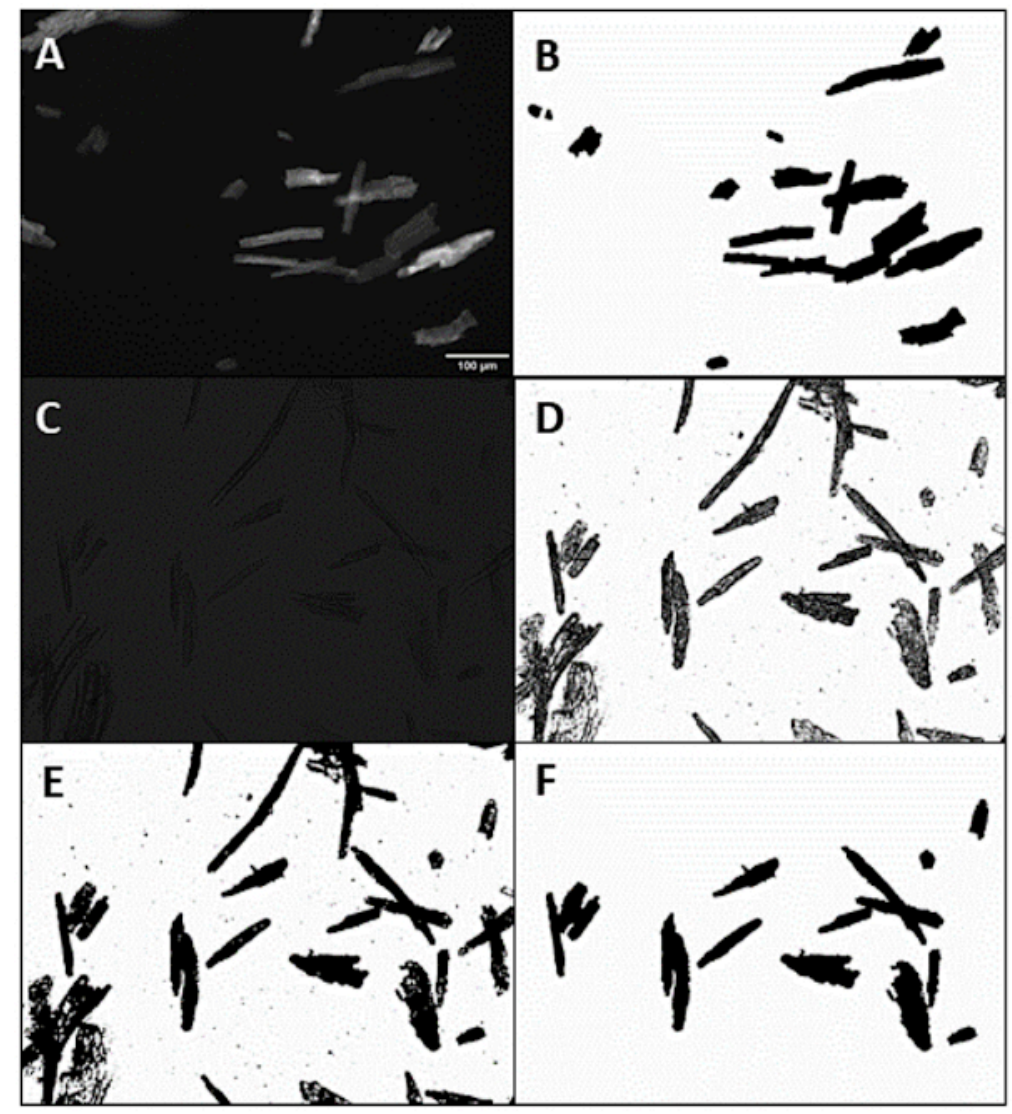

Figure 4: Strategy for cardiomyocyte segmentation. (A) Original fluorescent Troponin I stained cardiomyocyte image. (B) Triangle-thresholded image, after filling holes and excluding small objects and those touching the border. (C) Original bright field cardiomyocyte image (D) Edge-filtered and triangle-thresholded cardiomyocyte image (E) Edge-filtered image after morphological closing with a radius of two pixels (F) Same image after filling holes and excluding small objects and those touching the border. Scale bars $=100 \mu \mathrm{m}$. Please click here to view a larger version of this figure. 


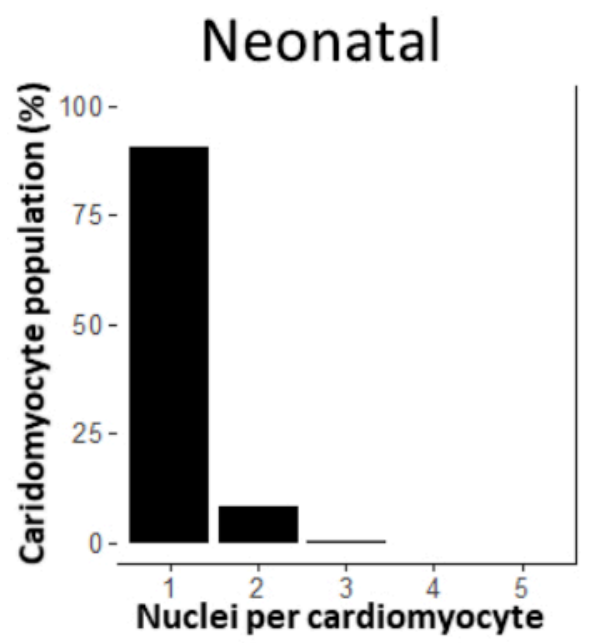

Juvenile

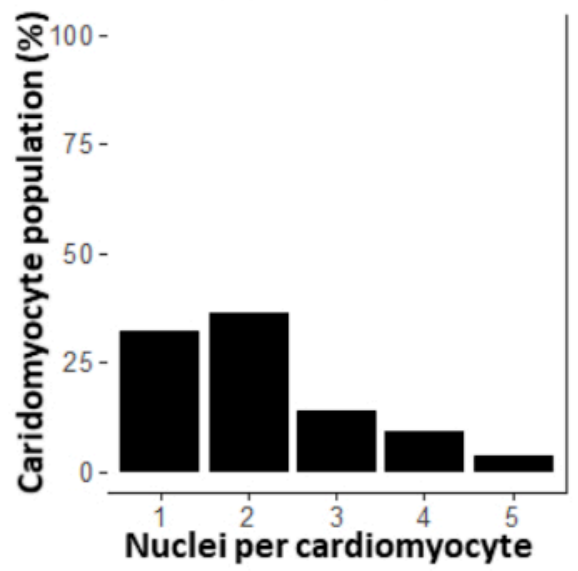

Figure 5: Classification of cardiomyocytes based on number of nuclei. Neonatal hearts (1 day old) contain more mononucleated cardiomyocytes than juvenile hearts (14 days old). Please click here to view a larger version of this figure. 

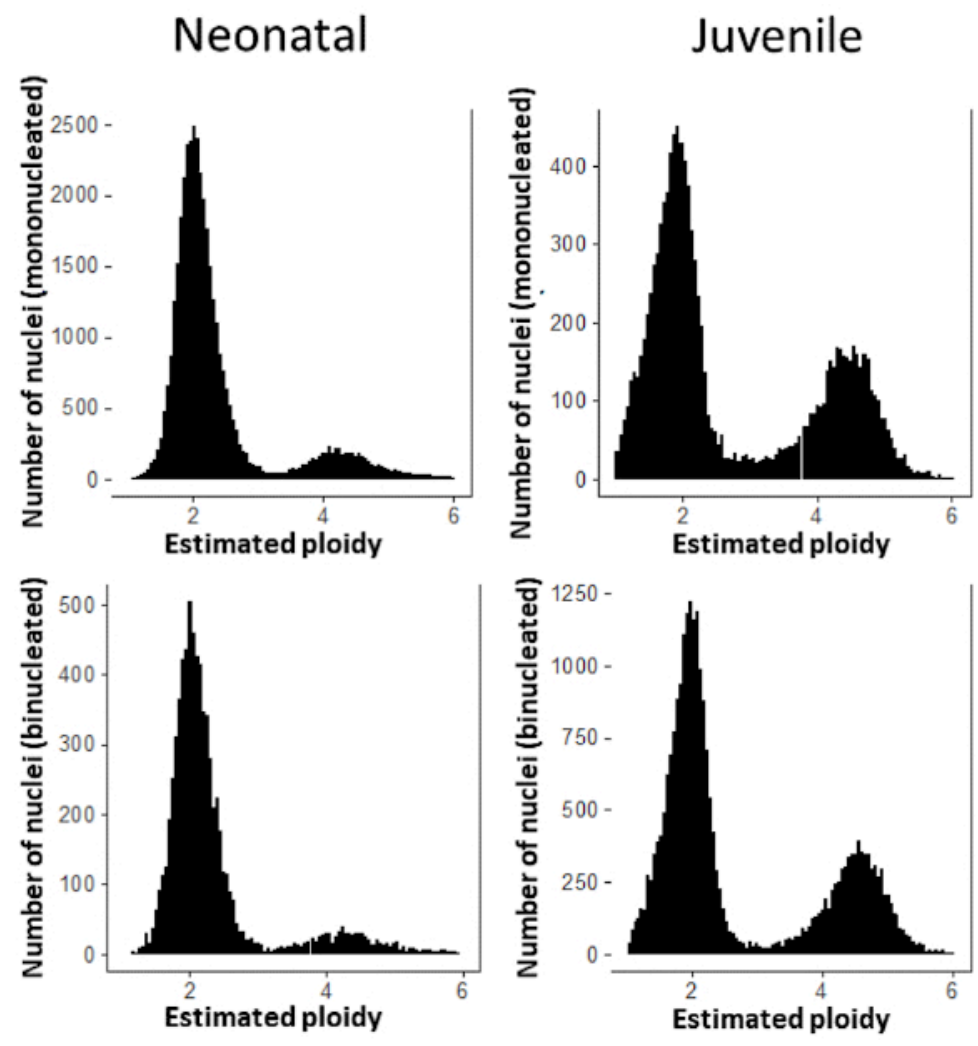

Figure 6: Distribution of cardiomyocyte DNA content per nucleus. In neonates (left), $13.5 \%$ of mononucleated CM nuclei are tetraploid and $11.9 \%$ of binucleated CM nuclei are tetraploid. In juveniles (right), $33.9 \%$ of mononucleated CM nuclei are tetraploid and $31.2 \%$ of binucleated CM nuclei are tetraploid. Please click here to view a larger version of this figure.

Supplementary File 1: Software Screenshots. Please click here to view this file (Right click to download).

Supplementary File 2: AnalyzeNucleation.py. Please click here to view this file (Right click to download).

Supplementary File 3: AnalyzeMultinucleatedServer.R. Please click here to view this file (Right click to download).

\section{Discussion}

Since cardiomyocytes cannot be maintained in culture, it is important to isolate primary cardiomyocytes to be able to study their architecture and function ${ }^{11}$. Hence, cardiomyocyte isolation techniques have been widely used in the cardiac field. If the goal is to determine functional aspects of cardiomyocytes, it is important to isolate viable cardiomyocytes. These live cardiomyocytes can also be used to perform immunostaining on isolated cardiomyocytes. However, optimizing the technique of isolating live cardiomyocytes is technically challenging, and even the best techniques typically only yield $60-65 \%$ live rodshaped cardiomyocytes, and the remaining cardiomyocytes are all balled up and dying or dead ${ }^{11,12}$. Here, we developed a technique that will allow researchers to first fix the heart, and then isolate cardiomyocytes efficiently. This new protocol allows for much higher yields of rod- 
shaped cardiomyocytes compared to previously published protocols. Furthermore, we developed an imaging analysis platform to categorize cardiomyocytes automatically based on nucleation and ploidy. With these new methodologies, groups can stain cardiomyocytes for different proteins, and study cardiomyocyte ploidy and nucleation status as surrogates for the regenerative potential of the heart.

The protocol described here is relatively straightforward, and can be performed without any advanced equipment. The amount of collagenase and incubation time for digestion might vary depending on the collagenase lot, and the company providing it. We used collagenase type 2 , since this is most widely used to digest the heart for obtaining live cardiomyocytes. Based on our observations, we determined that overnight incubation with $60 \mathrm{mg} / \mathrm{mL}$ collagenase type 2 is optimal for almost all mouse hearts regardless of the level of fibrosis. We have never had an issue of overdigestion as intracellular proteins are fixed and not as accessible as extracellular collagen. However, if the heart is not digested properly, more vigorous trituration might be needed, which causes cell fragmentation due to shear stress. Thus, it is crucial to make sure that the heart is digested properly before moving on to trituration. Stiffness of the heart can be tested by squeezing with forceps to assess the degree of digestion. Following incubation with collagenase, hearts should be less stiff and easy to tear apart. Other types of collagenase can also be used. A previous report used a combination of collagenases $B$ and $D^{14}$.

Furthermore, we believe that this protocol can be used to assess overall number of cardiomyocytes in the heart ${ }^{15}$. However, if the goal is to obtain and quantify all cardiomyocytes from the heart, it is important to incubate the hearts for extended periods of time in the collagenase solution (e.g., 3-7 days), where the collagenase solution should be replenished once a day. This will minimize inconsistencies in isolation efficiency by eliminating the impact of the degree of trituration on cardiomyocyte yield.

The use of DNA content to measure ploidy is not new, and has been used in flow cytometry for decades. Recently, it was shown that microscopy can similarly be used to estimate DNA content per nucleus ${ }^{16}$. Here, we implemented this strategy to measure ploidy of cardiomyocyte nuclei, as a surrogate for newly formed cardiomyocytes. The dogma in the field of cardiac regeneration is that only mononucleated, diploid cardiomyocytes can undergo cytokinesis and give rise to new cardiomyocytes. Since it is very challenging to measure new cardiomyocyte formation in vivo, isolating cardiomyocytes that have been chased after administration of a DNA nucleotide analog and determining the level of mononucleated, diploid cardiomyocytes has been used as an approximation of the ability of the heart to generate new cardiomyocytes $^{17}$. Here, we provide a macro for ImageJ that allows easy quantification of cardiomyocyte ploidy. At the very minimum, 500 nuclei must be measured to attain an accurate estimate of the location of the $\mathrm{G} 1$ peak. If care is taken to ensure that staining and imaging conditions are consistent across every well of the imaged plate, only 500 nuclei across the entire sample need to be imaged, otherwise, there need to be 500 nuclei per image group ${ }^{18,19}$. Limitations of imaging-based measurement of nucleation and ploidy include difficulty to distinguish nuclei from adherent cells from actual cardiomyocyte nuclei, when using two-dimensional images. Such adherent cells might result in overestimation of the quantity of multinucleated cells and decrease the accuracy of measurements of the tetraploid cardiomyocyte nucleus population. One possible strategy to solve this problem would be to use the cardiomyocyte nuclear marker $\mathrm{PCM}^{6,20}$. 
However, we have had difficulties to obtain reliable PCM1 staining on properly fixed cells or tissues.

Another potential limitation is that some nuclear stain images might have significant background cytoplasmic staining, preventing proper thresholding using Fiji's built in methods without extensive preprocessing. In addition, the irregular contribution of this background fluorescence into ploidy estimates reduces their accuracy. Moreover, if the cells are not left in DNA-staining solution for sufficient time, the fluorescent dye will not bind to saturation within the nuclei and the assumption of a linear relationship between nuclear integrated intensity and DNA content will no longer be accurate.

It should be noted that the software cannot segment cardiomyocyte clusters and instead removes them from analysis. Therefore, it is critically important to seed cardiomyocytes at a relatively low density (e.g., 1000 cells/ $\mathrm{cm}^{2}$ ). Further, the software cannot distinguish between two cardiomyocytes lined up end-to-end and long, singular cardiomyocytes. These sorts of clusters might erroneously inflate multinucleation estimates.

Although the described method does not allow for obtaining viable cardiomyocytes and thus cannot be used to measure dynamic cellular processes, if the goal is to perform immunostaining, we believe that the described method is superior to existing protocols with higher yields of cardiomyocytes and better quality in terms of morphology and protein localization. Finally, the described method could be used to isolate cardiomyocytes from clinical samples ${ }^{14,21}$. We believe the described methodology can help different researchers to obtain high-quality cardiomyocytes and measure nucleation and ploidy as surrogates for new cardiomyocyte formation.

\section{Disclosures}

The authors have nothing to disclose.

\section{Acknowledgments}

JHvB is supported by grants from the $\mathrm{NIH}$, Regenerative Medicine Minnesota, and an individual Biomedical Research Award from The Hartwell Foundation.

\section{References}

1. Benjamin, E. J. et al. Heart Disease and Stroke Statistics-2017 Update: A Report From the American Heart Association. Circulation. 135 (10), e146-e603 (2017).

2. Heidenreich, P. A. et al. Forecasting the future of cardiovascular disease in the United States: a policy statement from the American Heart Association. Circulation. 123 (8), 933-944 (2011).

3. Eschenhagen, T. et al. Cardiomyocyte Regeneration: A Consensus Statement. Circulation. 136 (7), 680-686 (2017).

4. Tzahor, E., Poss, K. D. Cardiac regeneration strategies: Staying young at heart. Science. 356 (6342), 1035-1039 (2017).

5. Bergmann, O. et al. Dynamics of Cell Generation and Turnover in the Human Heart. Cell. 161 (7), 1566-1575 (2015).

6. Richardson, G. D. Simultaneous Assessment of Cardiomyocyte DNA Synthesis and Ploidy: A Method to Assist Quantification of Cardiomyocyte Regeneration 
and Turnover. Journal of Visualized Experiments. (111) (2016).

7. Bergmann, O. et al. Evidence for cardiomyocyte renewal in humans. Science. 324 (5923), 98-102 (2009).

8. Senyo, S. E. et al. Mammalian heart renewal by preexisting cardiomyocytes. Nature. 493 (7432), 433-436 (2013).

9. Ang, K. L. et al. Limitations of conventional approaches to identify myocyte nuclei in histologic sections of the heart. American Journal of Physiology-Cell Physiology. 298 (6), C1603-1609 (2010).

10. Bergmann, O. et al. Identification of cardiomyocyte nuclei and assessment of ploidy for the analysis of cell turnover. Experimental Cell Research. 317 (2), 188-194 (2011).

11. O'Connell, T. D., Rodrigo, M. C., Simpson, P. C. Isolation and culture of adult mouse cardiac myocytes. Methods in Molecular Biology. 357 271-296 (2007).

12. Ackers-Johnson, M. et al. A Simplified, LangendorffFree Method for Concomitant Isolation of Viable Cardiac Myocytes and Nonmyocytes From the Adult Mouse Heart. Circulation Research. 119 (8), 909-920 (2016).

13. Shaklee, J. et al. Development of a Click-Chemistry Reagent Compatible with Mass Cytometry. Scientific Reports. 8 (1), 6657 (2018).

14. Mollova, M. et al. Cardiomyocyte proliferation contributes to heart growth in young humans. Proceedings of the National Academy of Sciences of the United States of America. 110 (4), 1446-1451 (2013).

15. Naqvi, N. et al. A proliferative burst during preadolescence establishes the final cardiomyocyte number. Cell. 157 (4), 795-807 (2014).
16. Roukos, V., Pegoraro, G., Voss, T. C., Misteli, T. Cell cycle staging of individual cells by fluorescence microscopy. Nature Protocols. 10 (2), 334-348 (2015).

17. Patterson, M. et al. Frequency of mononuclear diploid cardiomyocytes underlies natural variation in heart regeneration. Nature Genetics. 49 (9), 1346-1353 (2017).

18. Gomes, C. J., Harman, M. W., Centuori, S. M., Wolgemuth, C. W., Martinez, J. D. Measuring DNA content in live cells by fluorescence microscopy. Cell Division. 13, 6 (2018).

19. Woo, L. A. et al. High-content phenotypic assay for proliferation of human iPSC-derived cardiomyocytes identifies L-type calcium channels as targets. Journal of Molecular and Cellular Cardiology. 127, 204-214 (2018).

20. Morikawa, Y., Heallen, T., Leach, J., Xiao, Y., Martin, J. F. Dystrophin-glycoprotein complex sequesters Yap to inhibit cardiomyocyte proliferation. Nature. 547 (7662), 227-231 (2017).

21. Bergmann, O., Jovinge, S. Isolation of cardiomyocyte nuclei from post-mortem tissue. Journal of Visualized Experiments. (65) (2012). 